bysics Vol. 3, No. 1, pp. 1-16, 1967. Physics Publishing Co. Printed in Great Britain.

\title{
SATURATION OF CURRENT ALGEBRA EQUATIONS WITH HIGHER BARYONIC RESONANCES
}

\author{
R. GATTO, L. MAIANI* and G. PREPARATA $\dagger$ \\ Istituto di Fisica dell'Università, Firenze, Italy \\ (Received 15 March 1966)
}

\begin{abstract}
The saturation of the commutation relations of the chiral $U(3) \otimes U(3)$ algebra with supermultiplets of $\mathrm{SU}_{6}$ or $\mathrm{SU}_{6} \otimes \mathrm{O}_{3}$ is considered. The general helicity dependence of the matrix elements of axial charges at $\mathbf{p}=\infty$ is calculated. A continuity postulate is introduced to select the physically significant solutions from the multiplicity of solutions of the non-linear set of saturated commutator equations. A linex rized approach is developed to derive criteria of "connection" between two supermultiplets with respect to the chiral algebra and to calculate the renormalizations (at second order) of the couplings. The possible relevance of the connection criteria and of the compatibility with data of the coupling renormalizations to discriminate among various possibilities of classification of higher resonances is pointed out. Special cases discussed are classifications of negative parity resonances according to the representations and $\mathbf{5 6}$ of $\mathrm{SU}_{6}$ and $(\mathbf{2 0}, L=1)$ of $\mathrm{SU}_{6} \otimes \mathrm{O}_{3}$. Only the last one gives consistent predictions.
\end{abstract}

\section{Introduction}

HE recent work [1] by Adler and Weissberger has led to a calculation of the ratio $G_{A} / G_{V}$ from sum rule over pion-nucleon cross-sections derived from an equal time commutation relation beween non-strange axial generators of the chiral $U(3) \otimes U(3)$ algebra. Extension of their work o the strange generators has similarly led to sum-rules over $K$-nucleon cross-sections, allowng for a calculation of the $D / F$ ratio [2]. These calculations are based on the knowledge of xtrapolated empirical values of the pion-nucleon and $K$-nucleon cross-sections, inserted into he dispersive integrals appearing in the sum rules. A different approach is that used by Lee 3] and by Dashen and Gell-Mann [4], by saturating the commutation relations among stable articles and low-lying resonances and searching for a consistent solution of the obtained lgebraic system of equations.

In a preceding paper [5] we have followed the latter approach. We have saturated-the commutaIon relations of the chiral $U(3) \otimes U(3)$ algebra among the octet and decuplet baryon states

Istituto Superiore di Sanità, Roma, Italy.

Sezione di Firenze dell' Istituto Nazionale di Fisica Nucleare, Firenze, Italy. 
plus a set of resonant states selected on the basis of a previous classification of higher baryonic resonances [6]. The adopted classification of higher baryonic resonances of negative parity is based on the representation 20 of $S U_{6}$ with orbital angular momentum $L=1$ [6]. The states of 20 with $L=1$ (briefly $(20, L=1)$ ) are three unitary singlets with $J=1 / 2,3 / 2$, and $5 / 2$ and two unitary octets with $J=1 / 2$ and $3 / 2$.

In the saturation of the commutation relations the following set of states are thus included among the initial, final and intermediate states:

the states of 56 :

the states 20 with $L=1$ : one octet with $J^{P}=1 / 2^{+}$

one decuplet with $J^{P}=3 / 2^{+}$

one singlet and one octet with $J^{P}=1 / 2^{-}$

one singlet and one octet with $J^{P}=3 / 2^{-}$

one singlet with $J^{P}=5 / 2^{-}$.

In this note we present a more detailed discussion of the problem and consider its possible extensions. The extension of our results are made particularly simple by the use of the "linea ized approach" that we discuss in Section 4. The "linearized approach" consists in solving the commutator algebra equations perturbatively in the mixings between different supermultiplets. only solutions that can be obtained with continuity from the symmetric solution corresponding to absence of mixing are obtained by this procedure. We postulate that only these solutions have physical meaning (continuity postulate). Comparison with the particular case discussed of the mixing among 56 and $(20, L=1)$ shows that this is indeed the case. In Sections 2 and 3 we present a general procedure to write down the saturated commutator equations including states of any spin. We also consider in detail the solutions of the algebraic system in the case of 56 and $(20, L=1)$ and show that only two solutions may be a priori considered as physically possible. Only one is however admissible on the basis of the "continuity postulate" mentioned above.

\section{Saturation of the Commutation Rules}

Let us consider the commutation relation

$$
\left[F_{\mu}^{5}, F_{v}^{5}\right]=-\sqrt{3}\left(\begin{array}{lll}
8 & 8 & 8 \\
\mu & \nu & \lambda
\end{array}\right) F_{\lambda}
$$

between states with momentum $\mathbf{p}_{1}$ and $\mathbf{p}_{2}$ in the limit $\mathbf{p}_{1}=\mathbf{p}_{2}=\infty[7]$. In equation (1) $F_{\lambda}^{5}$ is an axial generator ( $\lambda$ denotes the set of $S U_{3}$ quantum numbers $I, I_{3}, Y$ ) of the chiral $U(3) \otimes U(3)$ and the symbol $\left(\begin{array}{lll}8 & 8 & 8 \\ \lambda & \mu & v\end{array}\right)$ denotes the relevant $S U_{3}$ Clebsch-Gordan coefficient. The saturatio of (1) requires the explicit evaluation of the limits for $\mathbf{p}_{1}=\mathbf{p}_{2} \rightarrow \infty$ of the matrix elements

$$
<J_{1}^{P_{1}}, h_{1}\left|F^{5}\right| J_{2}^{P_{2}}, h_{2}>
$$


here $<J_{1}^{P_{1}}, h_{1} \mid$ represents a baryon state with spin $J_{1}$ and parity $P_{1}$, with helicity $h_{1}$, gaken at momentum $\mathbf{p}_{1}$, and similarly for the state $\left|J_{2}^{P_{2}}, h_{2}\right\rangle$. In (2) we have for the moment aitted $S U_{3}$ indices. The axial generator $F^{5}$ is defined as

$$
F^{5}=\int d^{3} x J_{0}^{5}(\mathbf{x}, t)
$$

here $J_{\mu}^{5}(x)$ is the axial current. We note that the operator $F^{5}$ has the selection rule $\Delta h=0$, here $h$ is the helicity, and that the matrix elements between states of helicity $h$ and states of opposite helicity are related by the equation

$$
1
$$

$$
<J_{1}^{P_{1}}, h\left|F^{5}\right| J_{2}^{P_{2}}, h>=-P_{1} P_{2}<J_{1}^{P_{1}},-h\left|F^{5}\right| J_{2}^{P_{2}},-h>
$$

To obtain equation (3) one applies a parity operation followed by a $180^{\circ}$ rotation around an exis orthogonal to the momentum. From translation and rotation invariance we can also write the patrix element (2) in the form

$$
\left.<J_{1}^{P_{1}}, h_{1}\left|F^{5}\right| J_{2}^{P_{2}}, h_{2}>=(2 \pi)^{3} \delta^{3}\left(\mathbf{p}_{1}-\mathbf{p}_{2}\right) \delta_{h_{1} h_{2}}<J_{1}^{P_{1}}, h\left|J_{0}^{5}(\mathbf{0}, t)\right| J_{2}^{P_{2}, h}\right\rangle
$$

Where $h$ is the common value of $h_{1}$ and $h_{2}$. We are thus led to the calculation of the matrix element of the fourth component of an axial vector between two states of same momentum $p$ and spinparity $J_{1}^{P_{1}}$ and $J_{2}^{P_{2}}$. Such a matrix element will be a linear combination of all fourth components of axial vectors that can be constructed starting from the available covariants.

We shall use the Rarita-Schwinger formalism for particles of spin $J$. We describe a particle of $\operatorname{spin} J$ by a spinor with $J-1 / 2$ Lorentz indices $\mu, v, \ldots, \lambda$

$$
\psi_{\mu \nu \ldots \lambda} \ldots(p)
$$

ubject to the conditions:

(1) symmetry in the indices $\mu, v, \ldots, \lambda$ and zero trace

(2) transversality

$$
p^{\mu} \Psi_{\mu \nu .} \ldots \lambda(p)=0
$$

(3) orthogonality to $\gamma^{\mu}$

$$
\gamma^{\mu} \Psi_{\mu \nu . . \lambda}(p)=0
$$

(4) Dirac equation

$$
(p-m) \Psi_{\mu \nu} \ldots \lambda(p)=0
$$

rom the transformation properties of $\psi_{\mu \nu \ldots .}(p)$ under Lorentz transformations we can write in zeneral

$$
\Psi_{\mu .} \ldots \lambda(p)=\Lambda_{\mu}^{k}(p) \ldots \Lambda_{\lambda}^{h}(p) S(\Lambda) \Psi_{k} \ldots h(0)
$$


where: $\Lambda_{\mu} \nu(p)$ is the special Lorentz transformation which brings the four-vector $\tilde{p}=(\mathbf{o}, m)$ into the four-vector $p=(\mathbf{p}, E)$ i.e.

$$
p_{\mu}=\Lambda_{\mu}^{\nu}(p) \tilde{p}_{\nu}
$$

while $S(\Lambda)$ is the representation of the Lorentz transformation $\Lambda$ in spinor space. Explicitly

$$
S(\Lambda)=\sqrt{\frac{E+m}{2 m}}\left|\begin{array}{cr}
1 & \frac{\sigma \mathbf{p}}{E+m} \\
\frac{\sigma \mathbf{p}}{E+m} & 1
\end{array}\right|
$$

In equation (9) $\psi_{k} \ldots h(o)$ (with $h, \ldots, k=1,2,3$ ) is a four-component spinor of the form

$$
\psi_{k \ldots h}(o)=\left[\begin{array}{c}
X_{k} \ldots h \\
0
\end{array}\right]
$$

where $x_{k} \ldots h$ is a Pauli spinor and has the properties of being: symmetric and traceless in its indices $\ddot{k}_{\ldots h} \ldots h$ and orthogonal to the matrices $\sigma$, i.e. satisfying

$$
\sigma^{k} X_{k} \ldots h=0
$$

It is easy to verify that the representation (9) provides us with a wave function satisfying the required properties (1), (2), (3) and (4). In general any possible coupling takes the form

$$
\bar{\psi}^{\mu . . . \lambda\left(p_{1}\right) \Gamma_{\mu .} \ldots \sigma} \lambda_{, 05} \Phi_{\rho} \ldots \sigma\left(p_{2}\right)
$$

where $\psi_{\mu} \ldots \lambda\left(p_{1}\right)$ describes the particle of spin-parity $J_{1}^{P_{1}}$ and momentum $p_{1} \equiv\left(\mathbf{p}, E_{1}\right)$, while $\varphi_{\mathrm{p} .} \ldots \sigma\left(p_{2}\right)$ describes the particle of spin-parity $J_{2}^{P_{2}}$ and momentum $p_{2} \equiv\left(\mathbf{p}, E_{2}\right)$. The matrix $\Gamma_{\mu . \ldots \lambda, 05}^{\rho \ldots \sigma}$ is constructed from the Dirac matrices and from the available momenta in such a way that the covariant (14) behaves exactly as the fourth component of an axial vector. Explicit calculation of the covariants (14) involves the calculation of matrices of the kind

$$
S^{+}\left(\Lambda_{1}\right) \gamma_{4} \gamma_{R} S\left(\Lambda_{2}\right)
$$

where $\gamma_{R}$, with $R=1, \ldots, 16$ belongs to the set of independent Dirac matrices. Because of the form (12) of $\psi_{k} \ldots h(o)$, only the diagonal portion of (15) corresponding to the first two spinor indices is of relevance here. For the cases of interest to us we can limit ourselves to the evaluation of (15) for the following $\gamma_{R}: 1, \gamma_{4},-i \gamma_{4} \gamma_{5}$ and $i \gamma_{5}$. The form taken by the diagonal $2 \times 2$ portion of $S^{+}\left(\Lambda_{1}\right) \gamma_{4} \gamma_{R} S\left(\Lambda_{2}\right)$ mentioned before is:

$$
\frac{m}{\sqrt{m_{1} m_{2}}}
$$


for $\gamma_{R} \equiv 1$, where $m=1 / 2\left(m_{1}+m_{2}\right)$ is the average of the mass of the particle 1 and of the mass of particle 2;

$$
\stackrel{E}{\sqrt{m_{1} m_{2}}}
$$

for $\gamma_{R} \equiv \gamma_{4}$, where $E=\left|\mathbf{p}_{1}\right|=\left|\mathbf{p}_{2}\right|$ in the limit when these momenta tend to infinity;

$$
\frac{E}{\sqrt{m_{1} m_{2}}} \frac{\sigma \cdot \mathbf{p}}{|\mathbf{p}|}
$$

for $\gamma_{R} \equiv-i \gamma_{4} \gamma_{5} ;$ and

$$
\frac{\Delta m}{\sqrt{m_{1} m_{2}}} \frac{\boldsymbol{\sigma} \cdot \mathbf{p}}{|\mathbf{p}|}
$$

lor $\gamma_{R} \equiv i \gamma_{5}$, with $\Delta m=m_{1}-m_{2}$. Also of relevance are the contributions from expressions fo the kind

$$
q^{\mu} \psi_{\mu \ldots v}\left(p_{1}\right)=P \mu_{\psi_{\mu} \ldots v}\left(p_{1}\right)
$$

here $q^{\mu}=\left(p_{1}-p_{2}\right)^{\mu}$ and $P^{\mu}=\left(p_{1}+p_{2}\right)^{\mu}$. Equation $(20)$ holds by virtue of equation 6). Explicitly, choosing the space axis 3 in the direction of $\mathbf{p}$,

$$
q^{\mu} \Psi_{\mu \rho} \ldots \nu\left(p_{1}\right)=q^{\mu} \Lambda_{\mu}^{m}\left(p_{1}\right) \Lambda_{\rho}^{r}\left(p_{1}\right) \ldots \Lambda_{\nu}^{n}\left(p_{1}\right) S(\Lambda)\left[\begin{array}{c}
X_{m} r \ldots n \\
0
\end{array}\right]
$$

ind, in the limit $\mathbf{p}-\infty$, one has

$$
q^{\mu_{\psi_{\mu p} \ldots \nu} \ldots\left(p_{1}\right)=\frac{m}{m_{1}} \Delta m \Lambda_{\rho}^{r}\left(p_{1}\right) \ldots \Lambda_{\nu}^{n}\left(p_{1}\right) S(\Lambda)}\left[\begin{array}{c}
X_{3} r \ldots n \\
0
\end{array}\right]
$$

also note that, in the limit $\mathbf{p}-\infty$,

$$
\Lambda_{h}^{\lambda}\left(p_{1}\right) \Lambda_{\lambda}^{k}\left(p_{2}\right)-\delta_{h}^{k}+\frac{(\Delta m)^{2}}{2 m_{1} m_{2}} \delta_{3}^{k} \delta_{h}^{3}
$$

This result is useful whenever an index of one spinor is saturated with an index of the other spinor. We give now some examples of calculations of the couplings (14) (the spinors $X_{m} r \ldots n$ and $\xi_{m r \ldots n}$ refer to particles 1 and 2 respectively)

$\stackrel{1}{ \pm} \frac{1}{2}^{ \pm}$

$$
\begin{aligned}
& -i \bar{\psi} \gamma_{4} \gamma_{5} \phi-\frac{E}{\sqrt{m_{1} m_{2}}} x^{+} \sigma_{3} \xi \\
& -i \frac{P_{4}}{2 m} \bar{\psi} \gamma_{5} \phi-\frac{E}{\sqrt{m_{1} m_{2}}}\left(\frac{\Delta m}{m}\right) x^{+} \sigma_{3} \xi
\end{aligned}
$$


$\frac{1^{+}}{2} \longleftrightarrow \frac{1}{2}^{-}$

$$
\begin{aligned}
& \bar{\psi} \gamma_{4} \phi-\frac{E}{\sqrt{m_{1} m_{2}}} x^{+} \xi \\
& \frac{P_{4}}{2 m} \bar{\psi} \phi-\frac{E}{\sqrt{m_{1} m_{2}}} x^{+} \xi
\end{aligned}
$$

$\frac{1^{ \pm}}{2} \leftrightarrow \frac{3}{2}^{ \pm}$

$$
\bar{\psi}_{4} \phi-\frac{E}{\sqrt{m_{1} m_{2}}} x_{3}^{+} \xi
$$$$
\frac{q^{\mu}}{m} \bar{\psi}_{\mu \gamma_{4} \Phi}-\frac{E}{\sqrt{m_{1} m_{2}}}\left(\frac{\Delta m}{m}\right) x_{3}^{+} \xi
$$

$\frac{1^{+}}{2} \longleftrightarrow \frac{3}{2}^{ \pm}$

$$
-i \bar{\psi}_{4} \gamma_{5} \phi-\frac{E}{\sqrt{m_{1} m_{2}}} \frac{\Delta m}{\sqrt{m_{1} m_{2}}} x_{3}^{+} \sigma_{3} \xi
$$

$-i \frac{q^{\mu}}{m} \bar{\psi}_{\mu} \dot{\gamma}_{4} \gamma_{5} \Phi$ and $-i \frac{P_{4}}{2 m^{2}} q^{\mu_{\psi_{\mu} \gamma_{5}} \Phi}$ giving the same contribution

$\frac{1}{2}^{+} \leftarrow \frac{5^{-}}{2}$

$$
-i \frac{q^{\mu}}{m} \bar{\psi}_{\mu 4 \gamma_{5} \phi}-\frac{E}{\sqrt{m_{1} m_{2}}}\left(\frac{\Delta m}{m}\right)^{2}{\chi_{33} \sigma_{3} \xi}^{+}
$$

the other possible couplings giving the same contribution 
$\stackrel{ \pm}{\leftrightarrow} \frac{3^{ \pm}}{2}:$

$$
\begin{aligned}
& -i \bar{\psi}^{\lambda} \gamma_{4} \gamma_{5} \Phi \lambda-\frac{E}{\sqrt{m_{1} m_{2}}}\left[x_{h}^{+} \sigma_{3} \xi_{h}+\frac{1}{2} \frac{(\Delta m)^{2}}{m_{1} m_{2}} x_{3}^{+} \sigma_{3} \xi_{3}\right] \\
& -i \frac{q}{m} \bar{\psi} \gamma_{\gamma_{5} \phi_{4}}-\frac{E}{\sqrt{m_{1} m_{2}}} \frac{(\Delta m)^{2}}{m_{1} m_{2}} \chi_{3}^{+} \sigma_{3} \xi_{3}
\end{aligned}
$$

$\longleftrightarrow \frac{3-}{2}:$

$$
\begin{aligned}
& \bar{\psi}^{-\lambda} \gamma_{4} \Phi_{-}-\frac{E}{\sqrt{m_{1} m_{2}}}\left[\chi_{h}^{+} \xi_{h}+\frac{1}{2} \frac{(\Delta m)^{2}}{m_{1} m_{2}} x_{3}^{+} \xi_{3}\right] \\
& \frac{q^{\lambda}}{m} \bar{\psi} \lambda_{\lambda} \Phi_{4}-\frac{E}{\sqrt{m_{1} m_{2}}} \frac{\Delta m}{\sqrt{m_{1} m_{2}}} \chi_{3}^{+} \xi_{3}
\end{aligned}
$$

$\longrightarrow \frac{5-}{2}:$

$$
\begin{aligned}
& \bar{\psi}_{4 \lambda \Phi^{\lambda}}-\frac{E}{\sqrt{m_{1} m_{2}}}\left[x_{3 h}^{+} \xi_{h}+\frac{1}{2} \frac{(\Delta m)^{2}}{m_{1} m_{2}} x_{33}^{+} \xi_{3}\right] \\
& \frac{q^{\mu} q^{\nu}}{m^{2}} \bar{\psi}_{\mu \nu \Phi_{4}}-\frac{E}{\sqrt{m_{1 m_{2}}}}\left(\frac{\Delta m}{m}\right)^{2} x_{33}^{+} \xi_{3}
\end{aligned}
$$

lot all the possible covariants have been reported above. However the ones reported give all hose covariants which are linearly independent in the limit $\mathbf{p}-\infty$. To make the calculations easible we shall make two approximations:

(i) Inside each supermultiplet, in our case 56 and 20 with $L=1, \Delta m / m$ is neglected;

(ii) In the couplings between components of the different supermultiplets, i.e. in our case, In the couplings between 56 and $(20, L=1)$, only the lowest order couplings in $\Delta m / m$ are conidered. Under such assumptions we have the following dependences from the helicity of the hatrix elements of $F_{5}$ 


$$
\begin{aligned}
& <\frac{1}{2}^{ \pm}\left|F_{5}\right| \frac{1^{ \pm}}{2}>\propto \mathrm{x}^{+} \sigma_{3} \xi \\
& <\frac{3}{2}^{ \pm}\left|F_{5}\right| \frac{1}{2}^{\mp}>\propto \mathrm{x}_{3}^{+} \sigma_{3} \xi \\
& <\frac{1}{2}^{ \pm}\left|F_{5}\right| \frac{1}{2}^{\mp}>\propto x^{+} \xi \\
& <\frac{3^{ \pm}}{2}\left|F_{5}\right| \frac{3^{ \pm}}{2}>\propto \mathrm{x}_{k}^{+} \sigma_{3} \xi_{k} \\
& <\frac{1}{2}^{+}\left|F_{5}\right| \frac{5^{-}}{2}>\propto x^{+} \sigma_{3} \xi_{33} \\
& <\frac{3^{ \pm}}{2}\left|F_{5}\right| \frac{3}{2}^{\mp}>\infty x_{k}^{+} \xi_{k} \\
& <\frac{3}{2}^{ \pm}\left|F_{5}\right| \frac{1^{ \pm}}{2}>\propto \mathrm{x}_{3}^{+} \xi \\
& <\frac{5^{-}}{2}\left|F_{5}\right| \frac{3^{-}}{2}>\propto \mathrm{x}_{i 3}^{+} \xi_{i}
\end{aligned}
$$

Including now the $S U_{3}$ indices we write the states as $\left|K \rho, J^{P}{ }_{h}\right\rangle$, where the additional labels $R$ and $\rho$ denote respectively the dimensionality of the $S U_{3}$ representation and the set of quantum numbers $I, I_{3}$ and $Y$. The matrix elements of $J_{0 \lambda}^{5}(0, t)$ can be written as

$$
\begin{gathered}
<R_{1} \rho_{1}, J_{1}^{P_{1}} h_{1}\left|J_{o \lambda}^{5}(0, t)\right| R_{2} \rho_{2}, J_{2}^{P_{2}} h_{2}>=\frac{1}{(2 \pi)^{3}} \delta_{h_{1} h_{2}} C\left(J_{1}^{P_{1}} J_{2}^{P_{2}}, h\right) \mathbf{x} \\
\sum_{\xi} G_{\xi}\left(R_{1} R_{2} \mid J_{1}^{P_{1}} J_{2}^{P_{2}}\right)\left(\begin{array}{ccc}
R_{2} & 8 & R_{1} \xi \\
\rho_{2} & \lambda & \rho_{1}
\end{array}\right)
\end{gathered}
$$

where: $C\left(J_{1}^{P_{1}} J_{2}^{P_{2}}, h\right)$ are the values assumed by the couplings of our preceding discussion

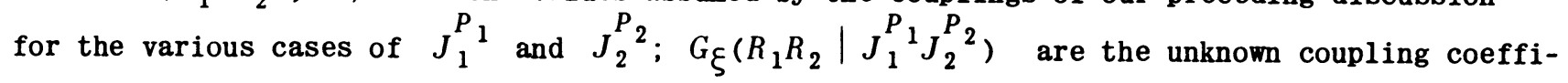
cients and the sum is extended over all the representations $R_{\xi}$ connected through the clebschGordan coefficient in equation (24). For $J_{1}^{P_{1}}=J_{2}^{P_{2}}=\frac{1^{+}}{2}$ our normalization coincides with that of Lee [4]. We note that from the property expressed by equation (3) the couplings $C\left(J_{1}^{P_{1}}, J_{2}^{P_{2}}, h\right)$ will satisfy

$$
C\left(J_{1}^{P}{ }_{1} J_{2}^{P_{2}}, h\right)=-P_{1} P_{2} C\left(J_{1}^{P_{1}} J_{2}^{P_{2}},-h\right)
$$

Also one has the symmetry relation

$$
C\left(J_{1}^{P_{1}} J_{2}^{P_{2}}, h\right)=C\left(J_{2}^{P_{2}} J_{1}^{P}, h\right)
$$


or the vector generators $F_{\nu}$ one can similarly write

$$
<R_{1} \rho_{1}, J_{1}^{P_{1}} h_{1}\left|F_{\nu}\right| R_{2} P_{2}, J_{2}^{P_{2}} h_{2}>=\delta^{3}\left(\mathbf{p}_{1}-\mathbf{p}_{2}\right) \delta_{J_{1} P_{J_{2}} P_{2} \delta_{R_{1} R_{2}} \delta_{h_{1} h_{2}} f\left(R_{1}\right)}\left(\begin{array}{cc}
R_{2} & 8 R_{1} a \\
\rho_{2} & v \rho_{1}
\end{array}\right)
$$

there for the octet representation $f(8)=\sqrt{3}$ and for the decuplet $f(\mathbf{1 0})=\sqrt{6}$. Substituting into squation (1), taken between states $\left|R_{1}, J_{1}^{P_{1}}\right\rangle$ and $\left|R_{2}, J_{2}^{P_{2}}\right\rangle$, we obtain the equation

$$
\begin{aligned}
& \sqrt{\frac{R_{1}}{Q}} \xi_{1}\left(\dot{R_{2}} R_{1} Q_{\beta}\right) \xi_{2}\left(R_{2} Q R_{1 \beta}\right) \xi_{3}\left(R_{2} R_{1} \dot{Q_{\beta}}\right)\left[1-\xi_{1}\left(88 Q_{\alpha}\right)\right] \times \\
& \sum T G_{\xi}\left(T R_{1} \mid j^{\pi} J_{1}^{P_{1}}\right) G_{\xi^{\prime}}\left(T R_{2} \mid j^{\pi} J_{2}^{P_{2}}\right)\left(T \xi^{\prime} \xi\left|\beta_{I I}\left(R_{1} 8 R_{2} 8\right)\right| Q \alpha \beta\right) \quad \mathbf{x} \\
& T \xi \xi^{\prime} j^{\pi} \\
& C\left(J_{1}^{P_{1}} j \pi, h\right) C\left(J_{2}^{P_{2}} j \pi, h\right)=-\sqrt{3} \delta_{Q 8} \delta_{\alpha a} \delta_{\beta a} \delta_{R_{1} R_{2}} \delta_{J_{1} P_{J_{2}} P_{2}} R_{1} f\left(R_{1}\right)
\end{aligned}
$$

In equation (28) the notations are the same as those used by de Swart [8]: in particular $\left(T \xi^{\prime} \xi\left|\beta_{I I}\left(R_{1} 8 R_{2} 8\right)\right| Q \alpha \beta\right)$ is a crossing matrix, the symbols $\xi_{1}\left(R_{2}^{*} R_{1} Q_{\beta}\right), \xi_{2}\left(R_{2} Q R_{1 \beta}\right)$, etc. are phase factors, $Q \propto \beta$ stands for $1,8_{a a}, 8_{a s}, 8_{s a}, 8_{s s}, 10,10^{*}, 27$. The values of $\left(R_{1} J_{1}^{P_{1}}\right),\left(R_{2}, J_{2}^{P_{2}}\right),(T, j \pi)$ vary over the set of baryon states selected to saturate the commutation relations.

\section{Solution of the Saturated Current Algebra Equations}

The compact form, equation (28), is general and applies to any problem of the kind we are discussing. Specialized to our case, with the particular choice of saturating states we have ade, it leads to a set of 49 non-linear equations to be satisfied by the unknown strengths $G_{\xi}\left(R_{1} R_{2} \mid J_{1}^{P_{1}} J_{2}^{P_{2}}\right)$. In spite of their apparent complication it is possible to solve the system of equations. We find essentially (i.e. apart from some choices of signs) two significant solutions (on the requirements that $D / F>o$ and $G\left(8,10 \mid 1 / 2^{+}, 3 / 2^{+}\right) \neq o$ ). The two solutions are reported in Table 1 . We recall that the strengths $G$ are all in principle susceptible of physical interpretations in terms of extrapolations of matrix elements of high energy neutrino processes, and in terms of their connection to strong couplings implied by Goldberger-Treiman arguments. 
TABLE 1

Solutions of the current-algebra equations, equation (28). Solution I implies the relation $-\frac{G_{A}}{G_{V}}=\frac{1}{3} \frac{D+F}{D-F}$, well satisfied experimentally. Solution II implies instead the relation $-\frac{G_{A}}{G_{V}}=\frac{1}{3} \frac{D+F}{2 F-D}$.

The strength for the transition between the states $J_{1}^{P_{1}}$ of the $S U_{3}$ representation $R_{1}$ and the states $J_{2} P_{2}$ of the representation $R_{2}$ (for $R_{1}=R_{2}=8$ the index $\xi$ distinguishes between symmetric $(s)$ and antisymmetric $(a)$ coupling is called $G_{\xi}\left(R_{1} R_{2} \mid J_{1}^{P_{1}} J_{2}^{P_{2}}\right)$. The solutions depend on two parameters, $a$ and $d$, subject to the inequalities $0 \leqslant a \leqslant 1$ and $4 / 5 \sqrt{3} \leqslant|d| \leqslant 4 \sqrt{3}$. The symbols $\varepsilon_{1} \varepsilon_{2} \varepsilon_{3}, \eta_{1} \eta_{2} \eta_{3}$ denote arbitrary factors \pm 1 . For $a=1$ the solutions reproduce well-known $S U_{6}$ results for the $1 / 2^{+}$octet and the $3 / 2^{+}$decuplet.

\begin{tabular}{|c|c|c|}
\hline$G_{a}\left(88 \mid \frac{1^{+}}{2} \frac{1}{2}^{+}\right)$ & $\frac{2}{\sqrt{3}} a$ & $\frac{2}{\sqrt{3}} a$ \\
\hline$G_{s}\left(88 \mid \frac{1^{+}}{2} \frac{1^{+}}{2}\right)$ & $\sqrt{\frac{5}{3}} \frac{1}{3}(1+2 a)$ & $\sqrt{\frac{5}{3}} \frac{4 a-1}{3}$ \\
\hline$G\left(8 \quad 10 \mid \frac{1^{+}}{2} \frac{3^{+}}{2}\right)$ & $\varepsilon_{2} 2 \sqrt{15 a}$ & $\varepsilon_{2} \sqrt{60 a}$ \\
\hline$G_{a}\left(88 \mid \frac{1^{+}}{2} \frac{3^{-}}{2}\right)$ & 0 & 0 \\
\hline$G_{s}\left(88 \mid \frac{1^{+}}{2} \frac{3^{-}}{2}\right)$ & $\frac{2}{3} \sqrt{10(1-a)}\left(-\varepsilon_{1} \varepsilon_{3}\right)$ & $\frac{2}{3} \sqrt{10(1-a)}\left(-\varepsilon_{1} \varepsilon_{3}\right)$ \\
\hline$G_{a}\left(88 \mid \frac{1^{+}}{2} \frac{1^{-}}{2}\right)$ & $\varepsilon_{1} \frac{2}{\sqrt{3}} \sqrt{a(1-a)}$ & $\varepsilon_{1} \frac{2}{\sqrt{3}} \sqrt{a(1-a)}$ \\
\hline$G_{s}\left(88 \mid \frac{1^{+}}{2} \frac{1^{-}}{2}\right)$ & $\varepsilon_{1} \frac{2}{3} \sqrt{\frac{5 a(1-a)}{3}}$ & $\varepsilon_{1} \frac{4}{3} \sqrt{\frac{5 a(1-a)}{3}}$ \\
\hline$G\left(18 \mid \frac{3^{-}}{2} \cdot \frac{1^{+}}{2}\right)$ & $\varepsilon_{1} \eta_{1} \eta_{2} 4 \sqrt{\frac{(1-a)\left(25 d^{2}-48\right)}{27 d^{2}-48}}$ & $\varepsilon_{1} \eta_{1} \eta_{2} 4 \sqrt{\frac{(1-a)\left(25 d^{2}-48\right)}{27 d^{2}-48}}$ \\
\hline$G\left(18 \mid \frac{1^{-}}{2} \frac{1^{+}}{2}\right)$ & $-\eta_{2} \varepsilon_{1} 4 d \sqrt{\frac{2(1-a)}{3\left(27 d^{2}-48\right)}}$ & $-\eta_{2} \varepsilon_{1} 4 d \sqrt{\frac{2(1-a)}{3\left(27 d^{2}-48\right)}}$ \\
\hline$G\left(\begin{array}{ll}10 & 10\end{array} \mid \frac{3^{+}}{2} \frac{3^{+}}{2}\right)$ & $\sqrt{6}$ & $\sqrt{6}$ \\
\hline
\end{tabular}




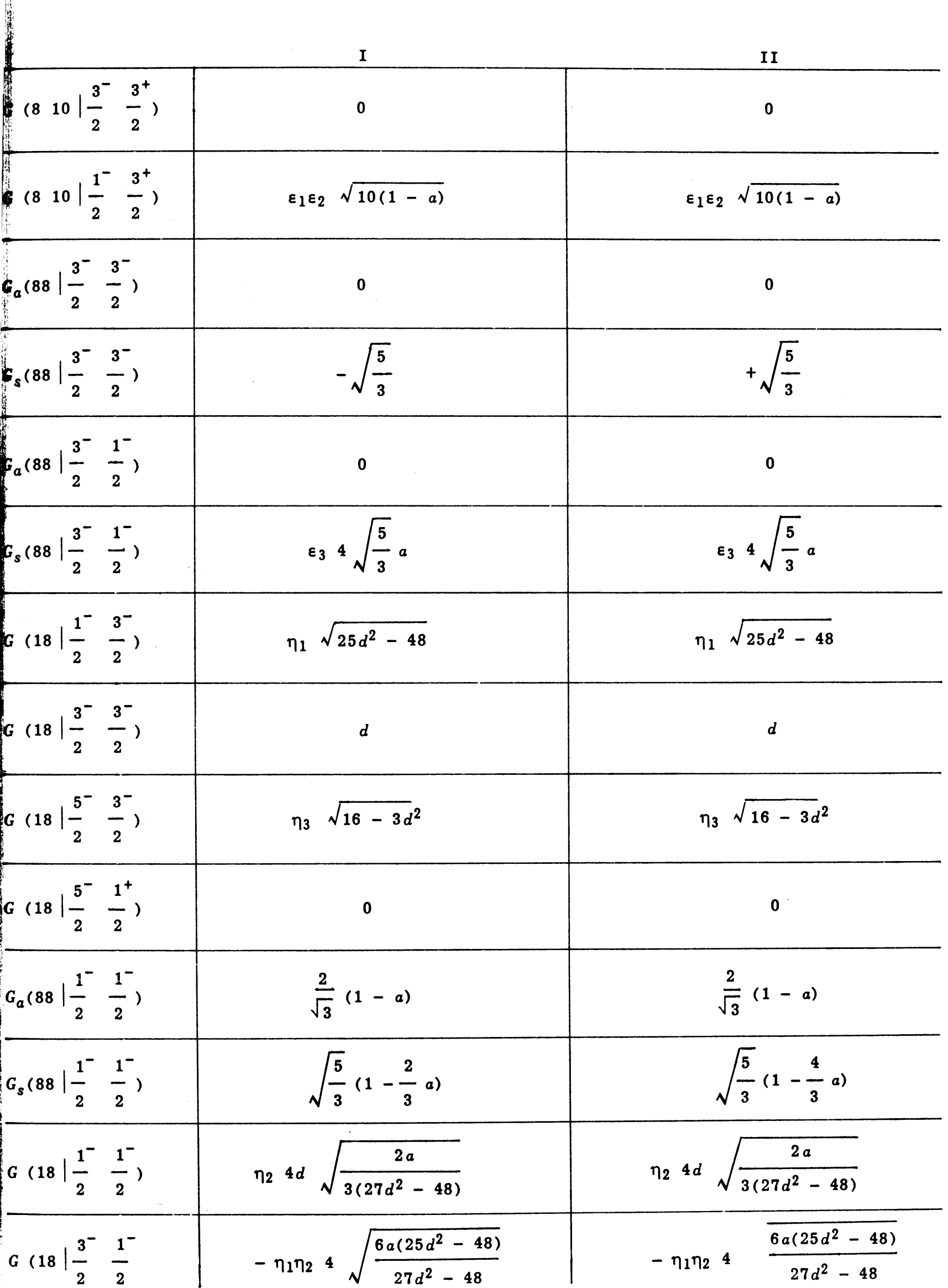


Of the two significant solutions, solution I agrees with experiment, while solution II disagrees. We note that in both solutions the important strengths $G_{a}\left(88 \mid 1 / 2^{+} 1 / 2^{+}\right)$and $G_{s}\left(88 \mid 1 / 2^{+} 1 / 2^{+}\right)$, which determine all octet-octet axial transitions depend on the single parameter $a$ (the other parameter $d$ only occurs in higher transitions). Eliminating this parameter and expressing the strengths in terms of the usual parameters $G_{A} / G_{V}$ and $D / F$ leads to the relations:

for solution I:

$$
-\frac{G_{A}}{G_{V}}=\frac{1}{3} \frac{D+F}{D-F}
$$

for solution II:

$$
-\frac{G_{A}}{G_{V}}=\frac{1}{3} \frac{D+F}{2 F-D}
$$

Furthermore the inequalities $0 \leqslant a \leqslant 1$ that must be satisfied by the parameter $a$ give for solution I:

$$
\frac{D}{F} \geqslant \frac{3}{2} \quad-\frac{G_{A}}{G_{V}} \leqslant \frac{5}{3}
$$

for solution II:

$$
\frac{D}{F} \leqslant \frac{3}{2} \quad-\frac{G_{A}}{G_{V}} \leqslant \frac{5}{3}
$$

We note that both solutions (29) and (30) contain the $S U_{6}$ limit

$$
\frac{D}{F}=\frac{3}{2} \quad-\frac{G_{A}}{G_{V}}=\frac{5}{3}
$$

However only solution I allows for $D / F \geqslant 3 / 2$, as suggested by the experiment. With $-G_{A} / G_{V}=1.2$ one obtains $D / F=1.76$, in agreement with experiment [9].

From Table 1 we can also obtain a relation between the $N^{*} N$ axial weak coupling at zero momentum transfer and $G_{A}$. Defining the $N^{*} N$ weak axial current as

$$
\frac{G}{\sqrt{2}} F_{1}^{A} \bar{\psi}_{\mu} \Psi+\ldots
$$

where $\psi_{\mu}$ denotes the $N^{*}$ and $\psi$ the nucleon, and we have neglected terms vanishing in the static 
limit, we find, for both solutions I and II

$$
F_{1}^{A}(o)^{2}=-\frac{4}{g}\left(3 \frac{G_{A}}{G_{V}}+1\right)
$$

hich gives for $-G_{A} / G_{V}=1.2$

$$
F_{1}^{A}(0)^{2}=1.16
$$

in good agreement with experiment.

\section{The Line arized Problem}

Both solutions I and II reproduce for $a=1$ the $S U_{6}$ results for the strengths $G$ limited to the multiplets of 56, i.e. for $(8,1 / 2)$ and for $(\mathbf{1 0}, 3 / 2)$. However only solution $I$ is capable, for limiting values of the parameters $a$ and $d$, to reproduce the complete $S U_{6}$ solution both for \$6 and for 20 with $L=1$, and, of course, with zero mixing between the two supermultiplets. The Belection in favor of solution I can thus be decided, on purely theoretical basis, from the requisite (continuity postulate), that it must continuously develop from the $S U_{6}$ symmetric solution by turning on the mixing parameters. Once we assume that the physical significant solution ust satisfy such a requisite we can think of an iterative scheme based on linearized equations, thich allows us to avoid the mathematical complexity due to the non-linearity of the problem. In general we shall proceed as follows. Suppose we saturate the current-algebra equations by a set of supermultiplets of $S U_{6}$ or $S U_{6} \otimes O_{3}$. We denote by $S_{1}, S_{2}, \ldots$ the different superultiplets. The left-hand side of a commutation relation like (1) can be written in block-form

$$
\left|\begin{array}{ll}
<S_{1}|\mathscr{C}| S_{1}> & <S_{1}|\mathscr{C}| S_{2}>\ldots \\
<S_{2}|\mathscr{C}| S_{1}> & <S_{2}|\mathscr{C}| S_{2}>\ldots
\end{array}\right|
$$

here

$$
<S_{i}|\mathscr{C}| S_{j}>
$$

denotes the submatrix obtained by taking the matrix element of the commutator $\mathscr{C}$ between the multiplets of $S_{i}$ and those of $S_{j}$. We now look for a solution infinitesimally close to the $S U_{6}$ solution. The non-diagonal elements $S_{i j}$ with $i \neq j$ of (33) are linear in the supposedly infinitesimal transition strengths between $S_{i}$ and $S_{j}$, as long as we neglect quadratic terms (including those leading from $S_{i}$ to $S_{k}$ with $k \neq i, j$ ). From the commutator equation we thus obtain, for the $i \longleftrightarrow j$ amplitudes a finite homogeneous linear set of equations, in the unknown strengths $i \leftarrow j$. Such a system may have non-trivial solution only if its rank is less than the number of unknowns. If this is the case we say that $S_{i}$ and $S_{j}$ are "connected", otherwise we say that they are "unconnected".

Consider now a diagonal submatrix $\left\langle S_{i}|\mathscr{C}| S_{i}>\right.$ and keep only terms linear in the deviation 
from the $S U_{6}$ solution (which we suppose to be well determined). The commutation equation imposes to these terms to be vanishing (this is a form of the non-renormalization theorem).

A deviation from $S U_{6}$ arises only if we include quadratic terms which arise in the saturation of the commutation relation with intermediate states of $S_{i}$ itself and of those $S_{j}$ "connected" to $S_{i}$. Inserting the solution for $i \hookleftarrow j$ transitions, again we obtain a linear system of equations, which, when solved, yields the renormalized $i \hookleftarrow i$ strengths.

If one applies this procedure restricting, as we have done above, to 56 and to $(20, L=1)$ we find that the linearized non diagonal subsystem for the transitions $(\mathbf{5 6}) \longleftrightarrow(\mathbf{2 0}, L=1)$ consists of 13 linear homogeneous equations in six unknowns. The rank of the system is five, leaving a single infinity of solutions depending on one parameter. Inserting these solutions in the. diagonal subsystems $56 \longleftrightarrow 56$ and $(20, L=1)$ one obtains the second order solution which is how ever sufficient to give the relation (29) between $G_{A} / G_{V}$ and $D / F$.

It is also important to note that the assumption (11) can be dropped in the linearized calcu. lation. The results are in fact unmodified when we allow for all couplings at any order in $\Delta \mathrm{m} / \mathrm{m}$ Application of the same procedure with restriction to 56 and to a negative parity 56 gives no non-trivial solution except $S U_{6}$. In fact these representations are easily seen to be "unconnected". Thus the mixing problems between 56 and another 56 multiplets of negative parity resonances has no solution, evolving with continuity from $S U_{6}$, except $S U_{6}$ itself. The simplicity of the linearized approach makes it easy to consider all similar cases.

\section{Conclusions and Outlooks}

A close analysis of the non-linear set of equations obtained from saturation of the chiral $U(3) \otimes U(3)$ commutation relations with the states of 56 and $(20, L=1)$, shows the existence of two solutions (reported on Table 1) passible of physical interpretation. The solutions depend on two undetermined parameters. Solution I leads to the relation

$$
-\frac{G_{A}}{G_{V}}=\frac{1}{3} \frac{D+F}{D-F} .
$$

and to the inequality $D / F \geqslant 3 / 2$, both satisfied by experiment. Solution II does not agree with data. The choice of solution I determines (apart from some ambiguities in sign) all the axial transition strengths among the stable baryon states of 56 and the resonant states of 20 with $L=1$, in terms of $D / F$ (or $G_{A} / G_{V}$ and another parameter).

The solutions depend on two undetermined parameters, related to the transition strengths between the two supermultiplets, in such a way that when these strengths are made vanishingly small one recovers the $S U_{6}$ solution for the 56 with no transition between the two supermultiplets. However only the empirically favored solution I reproduces, for vanishing mixings, the complete $S U_{6}$ solution both for 56 and $(20, L=1)$. The choice in favor of solution I could thus be decided, purely theoretically, from the requisite that only solutions which develop continuously from the $S U_{6}$ solution, when the mixing parameters are increased from zero value, are acceptable solutions. We call this requirement "continuity postulate". The assumption of such a postulate suggests a linearized approach to the problem consisting in solving by iteration the commutator equations for small mixing parameters. The linearized approach can be formulated in general terms leading to the notions of connected and unconnected supermultiplets (with 
pespect to the chiral algebra). For two supermultiplets $S_{i}, S_{j}$ the criterium of connectedness is given in terms of the rank of the determinant arising in the linearized set of equations obtained by restricting the saturated algebra to transitions from $S_{i}$ to $S_{j}$. The two supermultiplets are connected if the rank is less than the number of $S U_{3}$ independent amplitudes $S_{i} \leftrightarrow S_{j}$ : otherwise they are unconnected. The transition strengths inside the same supermultiplets are only renormalized at the second iteration. The renormalization is obtained by saturation of the commutation algebra between initial and final $S_{i}$, by only inclusion of connected intermediate $S_{j}$ (and of $S_{i}$ itself) up to second order in the mixing strengths.

The linearized approach, applied to the mixing problem of 56 and $(20, L=1)$ immediately reproduces solution $I$, at second order, giving in particular the relation (29) between $G_{A} / G_{V}$ and $D / F$. By the same procedure 56 is seen to be unconnected to a negative parity supermultiplet (56, $L=0)$. Classification of the negative parity baryonic resonances according to such a aultiplet is therefore less appealing than our assumed classification based on $(20, L=1)[6]$. This type of "indirect" application of current algebra may reveal itself very useful, and we are considering further extensions. The transition strengths between meson states can also be studied along the same lines. A discussion of higher meson resonances has led us to propose a classification according to the representation 35 with $L=1$ [10]. By saturating the $U(3) \otimes U(3)$ chiral algebra among the mesons of 35 and the mesons of 35 with $L=1$ one obtains verifiable predictions about the widths and branching ratios of the different modes of decay.

An interesting application concerns also the commutation relations of moments of local vector and axial currents satisfying the commutation relations of the chiral algebra.

The circumstance noted above, of the appearance of new parameters from the addition of a new set of saturating states, related to the transition strengths to such states, shows that no essential contradictions should arise when the set of saturating states is increased to include more and more states, unless the extension is made following wrong classification schemes. In principle one may then speculate of saturating the commutation relations in terms of representations of a non-compact group, except for the apparent mathematical complexities of such a problem.

\section{References}

1. S.L. ADLER, Phys. Rev. Letters 14, 1051 (1965); W. I. WEISSBERGER, Phys. Rev. Letters 14, 1047 (1965).

2. C.A. LEVINSON and I.J. MUZINICH, Phys. Rev. Letters 15, 715 (1965); D. AMATI, C. BOUCHIAT and J. NUYTS, Phys. Letters 19, 59 (1965); L.K. PANDIT and J. SCHECHTER, Phys. Let ters 19, 56 (1965).

3. B. W. LEE, Phys. Rev. Letters 14, 676 (1965).

4. R.F. DASHEN and M. GELL-MANN, Phys. Let ters 17, 145 (1965).

5. R. Gatto, L. maiani and G. PReparata, Phys. Rev. Letters (to be published).

6. R. Gatto, L. MaIANi and G. PREPARAta, Phys. Rev. 142, 1135 (1966). reported at the 0xford Conference on Elementary Particles, Oxford (September, 1965).

7. S. FUBINI and G. FURLAN, Physics 4, 229 (1965). 
8. J.J. De SWART, Nuovo Cimento 31, 420 (1964); see equation (7); J.J. De SWART, Rev. Mod. Phys. 35, 916 (1963).

9. H. COURANT et al., Phys. Rev. 136, B 1791. (1964).

10. E. BORCHI and R. GATTO, Phys. Letters 14, 352 (1965); R. GATTO, L. MAIANI and G. PREPARATA, Phys. Rev. 140, B 1579 (1965). 